\title{
Alternate-continuous-control systems with double-impulse
}

\author{
Xiang $\mathrm{Hu}^{1 *}$, Hongjuan $\mathrm{Wu}^{1}$, Yuming Feng ${ }^{2}$ and Jiang Xiong ${ }^{1}$
}

\author{
"Correspondence: \\ huyangyu0203@163.com \\ ${ }^{1}$ College of Computer Science and \\ Engineering, Chongqing Three \\ Gorges University, Wanzhou, \\ Chongqing 404100, P.R. China \\ Full list of author information is \\ available at the end of the article
}

\begin{abstract}
We propose a mathematical model that can control the stability of an unstable system. Periodicity is an important feature of the system. We add a continuous control to the first half of each period of the system and then add an impulse control $J 1$ at the $1 / 2$ period time. Again, we do not control the rest half of each period of the system. Finally, we add an impulse control $J 2$ at the end of each period of the system. The system is called an alternate-continuous-control system with double-impulse. We study the stability of the current system by constructing the Lyapunov function. Using the proposed method, we can control the Chua oscillator. The system has two impulse inputs per period, which is more in line with natural law than the system that only has a single-impulse input. Therefore, the system proposed in this paper is more practical than current mature control systems.
\end{abstract}

Keywords: alternate control; continuous control; double-impulse; Lyapunov function; index stability; Chua's oscillator; memristors

\section{Introduction}

At present, the stability of the nonlinear system control methods are: intermittent control [1-7], adaptive fuzzy control [8, 9], alternate control [10, 11], impulsive control [12-20], nonimpulsive control, continuous control, and so on [21-28] and so on. Our goal is a good system control. We will design some better control systems by studying the system control methods that are currently commonly used.

To make a nonlinear system stable, in this paper, first of all, each system period is divided into two equal parts. In the first part of the period, there are continuous inputs $C x(t)$, and in the other part, there is no input. We call it intermittent control system. Figure 1 provides the working principles of the intermittent control system. Next, we can add the impulsive control in the intermittent control system to control its better stability. We add an input of the impulsive $J 1$ to the system at the middle of each period. Similarly, we add an impulse $J 2$ at the end of each period of the system. In this way, there are a continuous control $C x(t)$ and impulsive controls $J 1$ and $J 2$ in each period of the system. We call it an alternate-continuous-control system with double-impulse. Figure 2 provides the working principles of alternate-continuous-control systems with double-impulse. The mathematical model proposed in this paper can be applied in many fields. In the medical field, it can be used in large-scale surgery to control patient's life characteristics. In the field of electronics, it can be used to control a variety of chaotic circuits, such as circuits that in-

(c) The Author(s) 2017. This article is distributed under the terms of the Creative Commons Attribution 4.0 International License (http://creativecommons.org/licenses/by/4.0/), which permits unrestricted use, distribution, and reproduction in any medium, provided you give appropriate credit to the original author(s) and the source, provide a link to the Creative Commons license, and indicate if changes were made. 
Figure 1 Working principle of the intermittent control system: in the first part of the period, there are continuous inputs $C_{x}(t)$, and in the other part, there is no input (we can write 0 ).

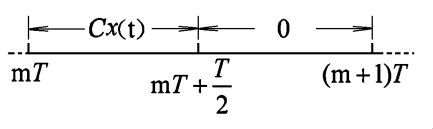

Figure 2 Alternate-continuous-control systems with double-impulse: in the first part of the period, there are continuous inputs $C_{x}(t)$, and in the other part, there is no input (we can write 0 ). We add an impulse $\lrcorner 1$ to the system at the middle of each period and an impulse $J 2$ at the end of each period.

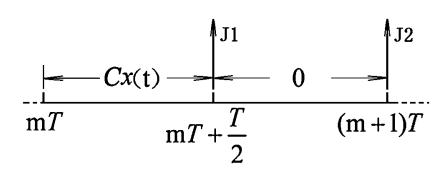

$(\mathrm{m}+1) T$

corporate memristors. In the field of intelligent robots, it can be used to control the robot walking gait in real time. It can also play a great advantage in the field of environmental pollution control [29-34].

In this paper, we construct alternate-continuous-control systems with double-impulse. We investigate the stability of the systems and get an exponential stability criterion in terms of a set of linear matrix inequalities. Some mathematical proofs make our conclusions reliable. At last, the Chua oscillator is controlled by using the results obtained.

The rest of this paper is organized as follows. In Section 3, we introduce some basic mathematical knowledge and mathematical symbols about system control, and two commonly used mathematical lemmas will be involved. In Section 4, we give the results of this paper and an exponential stability criterion, the main theory of this paper. In Section 5, we use this conclusion to control the Chua oscillator. Lastly, we give a summary of this paper.

\section{Problem formulation and preliminaries}

A classic nonlinear system can be described as

$$
\left\{\begin{array}{l}
\dot{x}(t)=H x(t)+f(x(t))+u(t), \\
x\left(t_{0}\right)=x_{0}
\end{array}\right.
$$

where $x \in R^{n}$ is a state vector, $H \in R^{n \times n}$ is a constant matrix, $f: R^{n} \rightarrow R^{n}$ is a continuous nonlinear function satisfying $f(0)=0$; we assume that there exists a diagonal matrix $L=$ $\operatorname{diag}\left(a_{1}, a_{2}, \ldots, a_{n}\right) \geq 0$ such that $\|f(x)\|^{2} \leq x^{T} L x$ for all $x \in R^{n} . H x(t)$ is the linear part of the system, $f(x(t))$ is the nonlinear interference, and $u(t)$ is the external input to system (1).

To stabilize the origin of system (1) by means of alternate-continuous-control systems with double-impulse, we impose two kinds of control, that is, assuming that the period of the system is $T$ and $m$ is a nonnegative integer, from $m T$ to $m T+\frac{T}{2}$, we set $u(t)=C x(t)$, where $C \in R^{n \times n}$ is a constant matrix, at time $m T+\frac{T}{2}$, an impulse $J 1$ given, from $m T+\frac{T}{2}$ to $(m+1) T$, no input to the system is given, and an impulse $J 2$ is given to the system at time $(m+1) T$.

So system (1) can be redefined as

$$
\begin{cases}\dot{x}(t)=H x(t)+f(x(t))+C x(t), & m T<t<m T+\frac{T}{2}, \\ x(t)=x\left(t^{-}\right)+J 1 x\left(t^{-}\right), & t=m T+\frac{T}{2}, \\ \dot{x}(t)=H x(t)+f(x(t)), & m T+\frac{T}{2}<t<(m+1) T, \\ x(t)=x\left(t^{-}\right)+J 2 x\left(t^{-}\right), & t=(m+1) T, \\ x\left(t_{0}\right)=x_{0}, & t_{0}=0,\end{cases}
$$


where $C, J 1, J 2 \in R^{n \times n}$ are constant matrices, and $T>0$ denotes the period of control.

We will use the following two mathematical lemmas.

Lemma 1 (Sanchez and Perez [35]) For any three real matrices $\Phi_{1}, \Phi_{2}, \Phi_{3}$ of appropriate dimensions and a scalar $\epsilon \geq 0$ such that $0<\Phi_{3}=\Phi_{3}^{T}$, we have the following inequality:

$$
\Phi_{1}^{T} \Phi_{2}+\Phi_{2}^{T} \Phi_{1} \leq \epsilon \Phi_{1}^{T} \Phi_{3} \Phi_{1}+\epsilon^{-1} \Phi_{2}^{T} \Phi_{3}^{-1} \Phi_{2}
$$

Lemma 2 (Boyd et al. [36]) The LMI

$$
\left[\begin{array}{ll}
W(x) & Z(x) \\
Z^{T}(x) & R(x)
\end{array}\right]>0,
$$

where $W(x)=W^{T}(x), R(x)=R^{T}(x)$, and $Z(x)$ depend affinely on $x$, is equivalent to

$$
R(x)>0, \quad W(x)-Z(x) R^{-1}(x) Z^{T}(x)>0 .
$$

We denote by $\lambda_{m}(D), \lambda_{M}(D)$, and $D^{T}$ the minimum eigenvalue, the maximum eigenvalue, and the transpose of a square matrix $D$, respectively. The Euclidean norm of the vector $x$ is denoted $\|x\|$. The matrix norm $\|\cdot\|$ is also referred to the Euclidean norm. We will use $D>0$ to display a symmetric positive definite matrix $D, D<0$ to display a symmetric negative definite matrix $D, D \leq 0$ to display a symmetric seminegative definite matrix $D$, and $D \geq 0$ to display a symmetric semi-positive definite matrix $D$. We denote $f\left(x\left(a^{-}\right)\right)=$ $\lim _{t \rightarrow a} f(x(t))$.

\section{Main results}

Theorem 1 Suppose that a symmetric and positive definite matrix $D \in R^{n \times n}$ and positive scalar constants $h_{1}>0, h_{2}>0, \epsilon_{1}>0$, and $\epsilon_{2}>0$ satisfy the following conditions:

(1) $D H+H^{T} D+D C+C^{T} D+\epsilon_{1} D^{2}+\epsilon_{1}^{-1} L+h_{1} D \leq 0$,

(2) $D H+H^{T} D+\epsilon_{2} D^{2}+\epsilon_{2}^{-1} L-h_{2} D \leq 0$,

(3) $h_{1} \frac{T}{2}-h_{2} \frac{T}{2}-\ln \lambda_{1}-\ln \lambda_{2}>0$,

where $\lambda_{1}=\lambda_{M}\left(D^{-1}(I+J 1)^{T} D(I+J 1)\right), \lambda_{2}=\lambda_{M}\left(D^{-1}(I+J 2)^{T} D(I+J 2)\right)$. Then the origin of system (2) is exponentially stable.

Proof First, we construct the Lyapunov function

$$
V(x(t))=x^{T}(t) D x(t)
$$

so that

$$
\lambda_{m}(D)\|x(t)\|^{2} \leq V(x(t)) \leq \lambda_{M}(D)\|x(t)\|^{2} .
$$

If $m T<t<m T+\frac{T}{2}$, then by (2), (3), and (4) we get

$$
\begin{aligned}
\dot{V}(x) & =2 x^{T} D \dot{x} \\
& =2 x^{T} D[H x+f(x)+C x]
\end{aligned}
$$




$$
\begin{aligned}
= & 2 x^{T} D H x+2 x^{T} D f(x)+2 x^{T} D C x \\
= & x^{T}[2 D H+2 D C] x+2 x^{T} D f(x) \\
= & x^{T}\left[D H+H^{T} D+D C+C^{T} D\right] x+2 x^{T} D f(x) \\
\leq & x^{T}\left[D H+H^{T} D+D C+C^{T} D\right] x \\
& +\epsilon_{1} x^{T} D^{2} x+\epsilon_{1}^{-1} x^{T} L x \\
= & x^{T}\left[D H+H^{T} D+D C+C^{T} D+\epsilon_{1} D^{2}+\epsilon_{1}^{-1} L\right] x \\
= & -h_{1} V(x)+x^{T}\left[D H+H^{T} D+D C+C^{T} D\right. \\
& \left.+\epsilon_{1} D^{2}+\epsilon_{1}^{-1} L+h_{1} D\right] x \\
\leq & -h_{1} V(x),
\end{aligned}
$$

where $D H+H^{T} D+D C+C^{T} D+\epsilon_{1} D^{2}+\epsilon_{1}^{-1} L+h_{1} D \leq 0$. We get

$$
V(x(t)) \leq V\left(x\left((m T)^{+}\right)\right) \exp \left(-h_{1}(t-m T)\right)
$$

where $m T<t<m T+\frac{T}{2}$.

$$
\text { If } t=m T+\frac{T}{2} \text {, then we get }
$$

$$
\begin{aligned}
\left.V(x)\right|_{t=m T+\frac{T}{2}} & =\left(x\left(t^{-}\right)+J 1 x\left(t^{-}\right)\right)^{T} D\left(x\left(t^{-}\right)+J 1 x\left(t^{-}\right)\right) \\
& =x\left(t^{-}\right)^{T}(I+J 1)^{T} D(I+J 1) x\left(t^{-}\right) \\
& \leq \lambda_{1} V\left(x\left(t^{-}\right)\right) .
\end{aligned}
$$

If $m T+\frac{T}{2}<t<(m+1) T$, then we get

$$
\begin{aligned}
D^{+} V(x)= & 2 x^{T} D \dot{x} \\
= & 2 x^{T} D[H x+f(x)] \\
= & 2 x^{T} D H x+2 x^{T} D f(x) \\
\leq & x^{T}\left[D H+H^{T} D\right] x+\epsilon_{2} x^{T} D^{2} x+\epsilon_{2}^{-1} x^{T} L x \\
= & x^{T}\left[D H+H^{T} D+\epsilon_{2} D^{2}+\epsilon_{2}^{-1} L\right] x \\
= & h_{2} V(x) \\
& +x^{T}\left[D H+H^{T} D+\epsilon_{2} D^{2}+\epsilon_{2}^{-1} L-h_{2} D\right] x \\
\leq & h_{2} V(x),
\end{aligned}
$$

where $D H+H^{T} D+\epsilon_{2} D^{2}+\epsilon_{2}^{-1} L-h_{2} D \leq 0$. We get

$$
V(x(t)) \leq \lambda_{1} V\left(x\left(\left(m T+\frac{T}{2}\right)^{-}\right)\right) \exp \left(h_{2}\left(t-m T-\frac{T}{2}\right)\right),
$$

where $m T+\frac{T}{2}<t<(m+1) T$. 


$$
\begin{aligned}
& \text { If } t=(m+1) T \text {, then we get } \\
& \qquad \begin{aligned}
\left.V(x)\right|_{t=(m+1) T} & =\left(x\left(t^{-}\right)+J 2 x\left(t^{-}\right)\right)^{T} D\left(x\left(t^{-}\right)+J 2 x\left(t^{-}\right)\right) \\
& =x\left(t^{-}\right)^{T}(I+J 2)^{T} D(I+J 2) x\left(t^{-}\right) \\
& \leq \lambda_{2} V\left(x\left(t^{-}\right)\right)
\end{aligned}
\end{aligned}
$$

We can do the following mathematical induction through (6), (7), (8), and (9).

Case 1: $m=0$.

Subcase 1 . If $0<t<\frac{T}{2}$, then we get

$$
V(x(t)) \leq V\left(x_{0}\right) \exp \left(-h_{1} t\right)
$$

So

$$
V\left(x\left(\frac{T^{-}}{2}\right)\right) \leq V\left(x_{0}\right) \exp \left(-h_{1} \frac{T}{2}\right)
$$

Subcase 2. If $\frac{T}{2} \leq t<T$, then we get

$$
\begin{aligned}
V(x(t)) & \leq \lambda_{1} V\left(x\left(\frac{T^{-}}{2}\right)\right) \exp \left(h_{2}\left(t-\frac{T}{2}\right)\right) \\
& \leq \lambda_{1} V\left(x_{0}\right) \exp \left(-h_{1} \frac{T}{2}+h_{2}\left(t-\frac{T}{2}\right)\right)
\end{aligned}
$$

and

$$
V\left(x\left(T^{-}\right)\right) \leq \lambda_{1} V\left(x_{0}\right) \exp \left(-h_{1} \frac{T}{2}+h_{2} \frac{T}{2}\right) .
$$

Subcase 3 . If $t=T$, then we get

$$
\begin{aligned}
V(x(T)) & \leq \lambda_{2} V\left(x\left(T^{-}\right)\right) \\
& \leq \lambda_{1} \lambda_{2} V\left(x_{0}\right) \exp \left(-h_{1} \frac{T}{2}+h_{2} \frac{T}{2}\right) .
\end{aligned}
$$

Case 2: $m=1$.

Subcase 1 . If $T<t<T+\frac{T}{2}$, then we get

$$
\begin{aligned}
V(x(t)) & \leq V\left(x\left(T^{+}\right)\right) \exp \left(-h_{1}(t-T)\right) \\
& \leq V(x(T)) \exp \left(-h_{1}(t-T)\right) \\
& \leq \lambda_{1} \lambda_{2} V\left(x_{0}\right) \exp \left(-h_{1}\left(t-\frac{T}{2}\right)+h_{2} \frac{T}{2}\right)
\end{aligned}
$$

and

$$
V\left(x\left(\left(T+\frac{T}{2}\right)^{-}\right)\right) \leq \lambda_{1} \lambda_{2} V\left(x_{0}\right) \exp \left(-h_{1} T+h_{2} \frac{T}{2}\right) .
$$


Subcase 2 . If $T+\frac{T}{2} \leq t<2 T$, then we get

$$
V(x(t)) \leq \lambda_{1}^{2} \lambda_{2} V\left(x_{0}\right) \exp \left(-h_{1} T+h_{2}(t-T)\right)
$$

So

$$
V\left(x\left((2 T)^{-}\right)\right) \leq \lambda_{1}^{2} \lambda_{2} V\left(x_{0}\right) \exp \left(-h_{1} T+h_{2} T\right) .
$$

Subcase 3. If $t=2 T$, then we get

$$
\begin{aligned}
V(x(2 T)) & \leq \lambda_{2} V\left(x\left((2 T)^{-}\right)\right) \\
& \leq \lambda_{1}^{2} \lambda_{2}^{2} V\left(x_{0}\right) \exp \left(-h_{1} T+h_{2} T\right) .
\end{aligned}
$$

Case 3: $m=2$.

Subcase 1. If $2 T<t<2 T+\frac{T}{2}$, then we get

$$
\begin{aligned}
V(x(t)) & \leq V(x((2 T))) \exp \left(-h_{1}(t-2 T)\right) \\
& \leq \lambda_{1}^{2} \lambda_{2}^{2} V\left(x_{0}\right) \exp \left(-h_{1}(t-T)+h_{2} T\right) .
\end{aligned}
$$

So

$$
V\left(x\left(\left(2 T+\frac{T}{2}\right)^{-}\right)\right) \leq \lambda_{1}^{2} \lambda_{2}^{2} V\left(x_{0}\right) \exp \left(-h_{1}\left(T+\frac{T}{2}\right)+h_{2} T\right) .
$$

Subcase 2 . If $2 T+\frac{T}{2} \leq t<3 T$, then we get

$$
V(x(t)) \leq \lambda_{1}^{3} \lambda_{2}^{2} V\left(x_{0}\right) \exp \left(-h_{1}\left(T+\frac{T}{2}\right)+h_{2}\left(t-\frac{3 T}{2}\right)\right) .
$$

So

$$
V\left(x\left((3 T)^{-}\right)\right) \leq \lambda_{1}^{3} \lambda_{2}^{2} V\left(x_{0}\right) \exp \left(-h_{1}\left(T+\frac{T}{2}\right)+h_{2} \frac{3 T}{2}\right) .
$$

Subcase 3 . If $t=3 T$, then we get

$$
V(x(3 T)) \leq \lambda_{1}^{3} \lambda_{2}^{3} V\left(x_{0}\right) \exp \left(-h_{1}\left(T+\frac{T}{2}\right)+h_{2} \frac{3 T}{2}\right)
$$

Case 4: $m=3$.

Subcase 1 . If $3 T<t<3 T+\frac{T}{2}$, then we get

$$
\begin{aligned}
V(x(t)) & \leq V(x((3 T))) \exp \left(-h_{1}(t-3 T)\right) \\
& \leq \lambda_{1}^{3} \lambda_{2}^{3} V\left(x_{0}\right) \exp \left(-h_{1}\left(t-\frac{3 T}{2}\right)+h_{2} \frac{3 T}{2}\right) .
\end{aligned}
$$

So

$$
V\left(x\left(\left(3 T+\frac{T}{2}\right)^{-}\right)\right) \leq \lambda_{1}^{3} \lambda_{2}^{3} V\left(x_{0}\right) \exp \left(-h_{1} 2 T+h_{2} \frac{3 T}{2}\right)
$$


Subcase 2. If $3 T+\frac{T}{2} \leq t<4 T$, then we get

$$
V(x(t)) \leq \lambda_{1}^{4} \lambda_{2}^{3} V\left(x_{0}\right) \exp \left(-h_{1} 2 T+h_{2}(t-2 T)\right)
$$

So

$$
V\left(x\left((4 T)^{-}\right)\right) \leq \lambda_{1}^{4} \lambda_{2}^{3} V\left(x_{0}\right) \exp \left(-h_{1} 2 T+h_{2} 2 T\right) .
$$

Subcase 3. If $t=4 T$, then we get

$$
V(x(4 T)) \leq \lambda_{1}^{4} \lambda_{2}^{4} V\left(x_{0}\right) \exp \left(-h_{1} 2 T+h_{2} 2 T\right) .
$$

Through the above induction, we get the following.

Case $k+1: m=k$.

Subcase 1. If $k T<t<k T+\frac{T}{2}$, then we get

$$
V(x(t)) \leq \lambda_{1}^{k} \lambda_{2}^{k} V\left(x_{0}\right) \exp \left(-h_{1}\left(t-\frac{k T}{2}\right)+h_{2} \frac{k T}{2}\right) .
$$

Subcase 2. If $k T+\frac{T}{2} \leq t<(k+1) T$, then we get

$$
V(x(t)) \leq \lambda_{1}^{k+1} \lambda_{2}^{k} V\left(x_{0}\right) \exp \left(-h_{1} \frac{(k+1) T}{2}+h_{2}\left(t-\frac{(k+1) T}{2}\right)\right) .
$$

Subcase 3. If $t=(k+1) T$, then we get

$$
\left.V(x(t))\right|_{t=(k+1) T} \leq \lambda_{1}^{k+1} \lambda_{2}^{k+1} V\left(x_{0}\right) \exp \left(-h_{1} \frac{(k+1) T}{2}+h_{2} \frac{(k+1) T}{2}\right) .
$$

From (10) we get that if $k T<t<k T+\frac{T}{2}$, then we let $t=k T$, so that

$$
\begin{aligned}
V(x(t)) & \leq \lambda_{1}^{k} \lambda_{2}^{k} V\left(x_{0}\right) \exp \left(-h_{1}\left(t-\frac{k T}{2}\right)+h_{2} \frac{k T}{2}\right) \\
& \leq \lambda_{1}^{k} \lambda_{2}^{k} V\left(x_{0}\right) \exp \left(-h_{1} \frac{k T}{2}+h_{2} \frac{k T}{2}\right) \\
& \leq \exp \left(k \ln \lambda_{1}+k \ln \lambda_{2}\right) V\left(x_{0}\right) \exp \left(-h_{1} \frac{k T}{2}+h_{2} \frac{k T}{2}\right) \\
& \leq V\left(x_{0}\right) \exp \left(-\left(h_{1} \frac{T}{2}-h_{2} \frac{T}{2}-\ln \lambda_{1}-\ln \lambda_{2}\right) k\right)
\end{aligned}
$$

for $k T<t<k T+\frac{T}{2}$.

From (11) we get that if $k T+\frac{T}{2} \leq t<(k+1) T$, then we let $t=(k+1) T$, so that

$$
\begin{aligned}
V(x(t)) & \leq \lambda_{1}^{k+1} \lambda_{2}^{k} V\left(x_{0}\right) \exp \left(-h_{1} \frac{(k+1) T}{2}+h_{2}\left(t-\frac{(k+1) T}{2}\right)\right) \\
& \leq \lambda_{1}^{k+1} \lambda_{2}^{k} V\left(x_{0}\right) \exp \left(-h_{1} \frac{(k+1) T}{2}+h_{2} \frac{(k+1) T}{2}\right)
\end{aligned}
$$




$$
\begin{aligned}
& \leq V\left(x_{0}\right) \exp \left(-h_{1} \frac{(k+1) T}{2}+h_{2} \frac{(k+1) T}{2}+(k+1) \ln \lambda_{1}+k \ln \lambda_{2}\right) \\
& \leq V\left(x_{0}\right) \exp \left(-h_{1} \frac{T}{2}-h_{1} \frac{k T}{2}+h_{2} \frac{k T}{2}+h_{2} \frac{T}{2}+\ln \lambda_{1}+k \ln \lambda_{1}+k \ln \lambda_{2}\right) \\
& \leq V\left(x_{0}\right) \exp \left(-h_{1} \frac{T}{2}+h_{2} \frac{T}{2}+\ln \lambda_{1}-\left(h_{1} \frac{T}{2}-h_{2} \frac{T}{2}-\ln \lambda_{1}-\ln \lambda_{2}\right) k\right)
\end{aligned}
$$

for $k T+\frac{T}{2} \leq t<(k+1) T$.

From (12) we get that, for $t=(k+1) T$,

$$
\begin{aligned}
\left.V(x(t))\right|_{t=(k+1) T} & \leq \lambda_{1}^{k+1} \lambda_{2}^{k+1} V\left(x_{0}\right) \exp \left(-h_{1} \frac{(k+1) T}{2}+h_{2} \frac{(k+1) T}{2}\right) \\
& \leq \exp \left((k+1) \ln \lambda_{1}+(k+1) \ln \lambda_{2}-h_{1} \frac{(k+1) T}{2}+h_{2} \frac{(k+1) T}{2}\right) V\left(x_{0}\right) \\
& \leq V\left(x_{0}\right) \exp \left(-\left(h_{1} \frac{T}{2}-h_{2} \frac{T}{2}-\ln \lambda_{1}-\ln \lambda_{2}\right)(k+1)\right)
\end{aligned}
$$

From (13), (14), (15), and the conditions of Theorem 1 we conclude that $k \rightarrow \infty$ as $t \rightarrow \infty$. So

$$
\lim _{t \rightarrow \infty} V(x(t))=0
$$

which ends the proof.

Corollary 1 As a consequence of Lemma 2, the first two conditions of Theorem 1 are equivalent to the following two LIMs:

$$
\begin{aligned}
& {\left[\begin{array}{cc}
D H+H^{T} D+D C+C^{T} D+\epsilon_{1}^{-1} L+h_{1} D & -D \\
-D & -\epsilon_{1}^{-1} I
\end{array}\right] \leq 0} \\
& {\left[\begin{array}{cc}
D H+H^{T} D+\epsilon_{2}^{-1} L-h_{2} D & -D \\
-D & -\epsilon_{2}^{-1} I
\end{array}\right] \leq 0 .}
\end{aligned}
$$

\section{Numerical example}

Studying system examples, we can define the original and dimensionless form of Chua's oscillator [37] as follows:

$$
\left\{\begin{array}{l}
\dot{x}_{1}=\omega\left(x_{2}-x_{1}-g\left(x_{1}\right)\right) \\
\dot{x}_{2}=x_{1}-x_{2}+x_{3} \\
\dot{x}_{3}=-\gamma x_{2}
\end{array}\right.
$$

where $\omega$ and $\gamma$ are two parameters, $g(x)$ is a piecewise linear characteristic of Chua's diode. It can be defined by

$$
g(x)=b x+0.5(a-b)(|x+1|-|x-1|)
$$

where $a$ and $b$ are two constants such that $0>b>a$. 


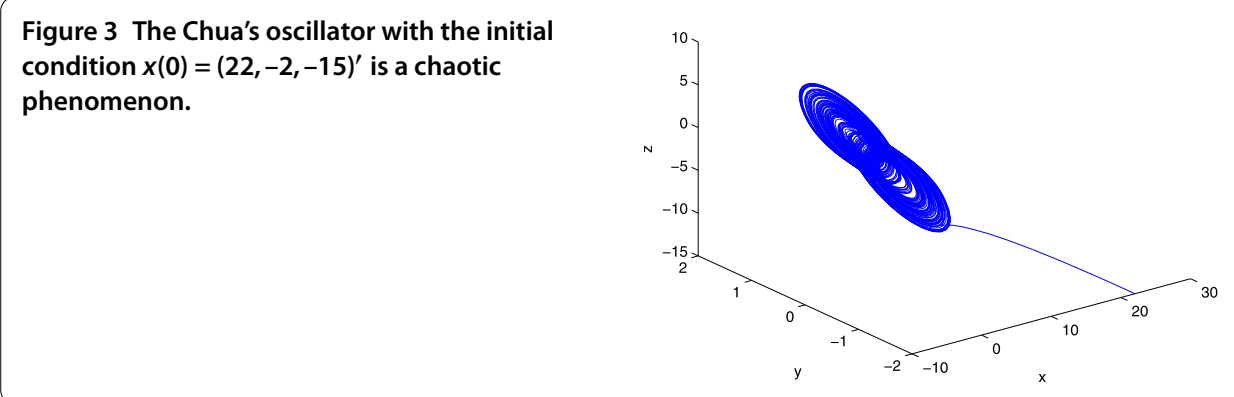

Next, we choose the parameters $\omega=9.1196, \gamma=15.8946, a=-1.44905$, and $b=$ -0.79735 , which make Chua's circuit (18) chaotic [37]. Figure 3 shows that Chua's oscillator with the initial condition $x(0)=(22,-2,-15)^{\prime}$ is a chaotic phenomenon.

We can redefine system (18) as follows:

$$
\dot{\mathbf{x}}=H \mathbf{x}+f(\mathbf{x}),
$$

where

$$
H=\left[\begin{array}{ccc}
-\omega-\omega b & \omega & 0 \\
1 & -1 & 1 \\
0 & -\gamma & 0
\end{array}\right]
$$

and

$$
f(x)=\left[\begin{array}{c}
-0.5 \omega(a-b)\left(\left|x_{1}+1\right|-\left|x_{1}-1\right|\right) \\
0 \\
0
\end{array}\right] .
$$

So we get

$$
\begin{aligned}
\|f(x)\|^{2} & =0.25 \omega^{2}(a-b)^{2}\left[\left(x_{1}+1\right)^{2}+\left(x_{1}-1\right)^{2}-2\left|\left(x_{1}+1\right)\left(x_{1}-1\right)\right|\right] \\
& =0.5 \omega^{2}(a-b)^{2}\left(x_{1}^{2}+1-\left|x_{1}^{2}-1\right|\right) \\
& = \begin{cases}\omega^{2}(a-b)^{2}, & x_{1}^{2}>1, \\
\omega^{2}(a-b)^{2} x_{1}^{2}, & x_{1}^{2} \leq 1\end{cases} \\
& \leq \omega^{2}(a-b)^{2} x_{1}^{2} .
\end{aligned}
$$

Thus we set $L=\operatorname{diag}\left(\omega^{2}(a-b)^{2}, 0,0\right)$ and choose

$$
\begin{aligned}
C & =\operatorname{diag}(-49,-42,-32), \\
J 1 & =\left[\begin{array}{ccc}
-1 & 0 & -0.5 \\
0 & 0.2 & 0.4 \\
1.5 & 0 & 0.5
\end{array}\right], \\
J 2 & =\left[\begin{array}{ccc}
-1 & 0 & -0.8 \\
0 & 0.3 & 0.9 \\
1.8 & 0 & 1.5
\end{array}\right] .
\end{aligned}
$$




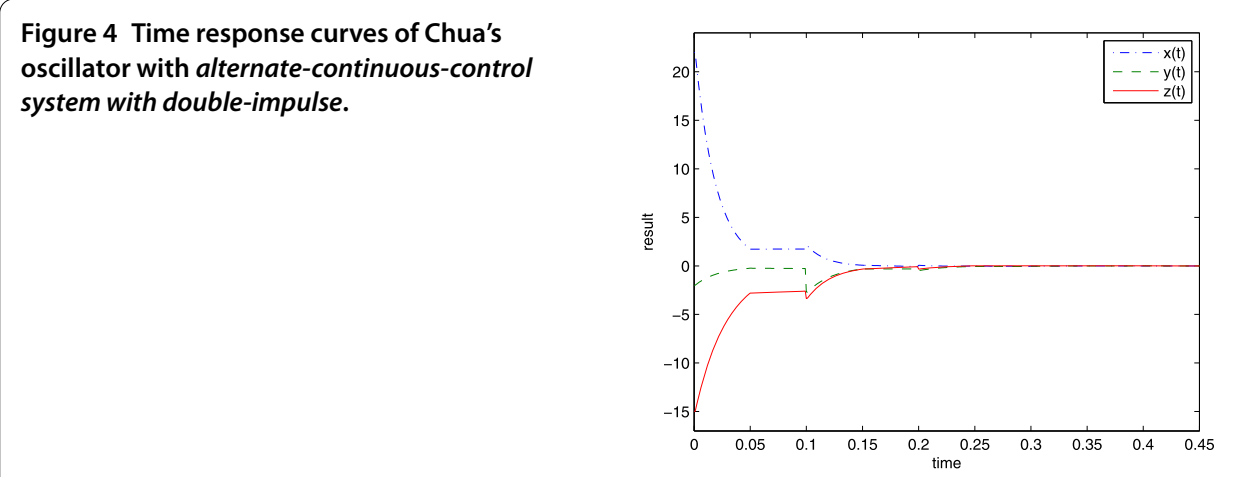

With $T=0.10$, solving LMIs (16) and (17) and the inequality $h_{1} \frac{T}{2}-h_{2} \frac{T}{2}-\ln \lambda_{1}-\ln \lambda_{2}>0$, we obtain a feasible solution

$$
\epsilon_{1}=0.5, \quad \epsilon_{2}=0.5, \quad h_{1}=21, \quad h_{2}=20,
$$

and

$$
D=\left[\begin{array}{ccc}
3.7422 & 1.3317 & 0.4360 \\
1.3317 & 3.8921 & -0.4290 \\
0.4360 & -0.4290 & 2.1051
\end{array}\right]
$$

Thus, by Theorem 1 we know that the origin of system (18) becomes exponentially stable. The time response curves of Chua's oscillator with the proposed method is shown in Figure 4.

\section{Conclusions}

We proposed a new model of a control system named an alternate-continuous-control system with double-impulse. The introduction of an impulse input has played a positive role in the stability control of the system. Theorem 1 gives stability criteria of the current new system control. Moreover, the chaotic Chua circuit can be controlled by the new method.

Through the control method of this paper, we can control most of the nonlinear systems. The method can be used in physics, electronics, robotics, and other fields. Later, we can design more control methods to stabilize nonlinear systems by combining impulse control and intermittent control.

Funding

This work is supported by Research Foundation of Chongqing Municipal Education Commission (KJ1710253) and Chongqing Municipal Key Laboratory of Institutions of Higher Education (Grant No. C16).

Competing interests

The authors claim that they have no competitive interest.

Authors' contributions

YF has provided the main idea of the paper. XH has proved the main results and prepared the paper by $4 T E X$. HW and JX have provided some figures. All authors read the paper and approved the final version.

\section{Author details}

'College of Computer Science and Engineering, Chongqing Three Gorges University, Wanzhou, Chongqing 404100, P.R. China. ${ }^{2}$ Key Laboratory of Intelligent Information Processing and Control, Chongqing Three Gorges University, Wanzhou, Chongqing 404100, P.R. China. 


\section{Publisher's Note}

Springer Nature remains neutral with regard to jurisdictional claims in published maps and institutional affiliations.

\section{Received: 22 May 2017 Accepted: 26 August 2017 Published online: 25 September 2017}

\section{References}

1. Chen, Q, Wang, X, Wan, H, Yang, R: A logic circuit design for perfecting memristor-based material implication. IEEE Trans. Comput.-Aided Des. Integr. Circuits Syst. 36(2), 279-284 (2017)

2. Li, C, Feng, G, Liao, X: Stabilization of nonlinear systems via periodically intermittent control. IEEE Trans. Circuits Syst. II, Express Briefs 54(11), 1019-1023 (2007)

3. Huang, J, Li, C, He, X: Stabilization of a memristor-based chaotic system by intermittent control and fuzzy processing. Int. J. Control. Autom. Syst. 11(3), 643-647 (2013)

4. Huang, J, Li, C, Han, Q: Quasi-synchronization of chaotic neural networks with parameter mismatch by periodically intermittent control. In: Computer Science and Information Engineering, 2009 WRI World Congress on, Los Angeles, California, USA, 2009, March 31-April 2 (2009)

5. Huang, T, Li, C, Liu, X: Synchronization of chaotic systems with delay using intermittent linear state feedback. Chaos $18,033122(2008)$

6. Yang, X, Feng, Z, Feng, J, Cao, J: Synchronization of discrete-time neural networks with delays and Markov jump topologies based on tracker information. Neural Netw. 85, 157-164 (2017)

7. Yang, X, Cao, J, Liang, J: Exponential synchronization of memristive neural networks with delays: interval matrix method. IEEE Trans. Neural Netw. Learn. Syst. doi:10.1109/TNNLS.2016.2561298

8. Yin, S, Yu, H, Shanazi, R, Haghani, A: Fuzzy adaptive tracking control of constrained nonlinear switched stochastic pure-feedback systems. IEEE Trans. Cybern. (2017). doi:10.1109/TCYB.2016.2521179

9. Chen, L, Liu, W, Hou, Z, Huang, T, Yu, J, Tan, M: An adaptive Takagi-Sugeno fuzzy model based predictive controller for piezoelectric actuator. IEEE Trans. Ind. Electron. 64, 3048-3058 (2017)

10. Wang, J, Wu, H, Huang, T: Passivity-based synchronization of a class of complex dynamical networks with time-varying delay. Automatica 56, 105-112 (2015)

11. Huang, T, Li, C, Duan, S, Starzyk, JA: Robust exponential stability of uncertain delayed neural networks with stochastic perturbation and impulse effects. IEEE Trans. Neural Netw. Learn. Syst. 23(6), 866-875 (2012)

12. Feng, Y, Li, C, Huang, T: Sandwich control systems with impulse time windows. Int. J. Mach. Learn. Cybern. (2016). doi:10.1007/s13042-016-0580-5

13. Feng, Y, Li, C, Huang, T: Periodically multiple state-jumps impulsive control systems with impulse time windows. Neurocomputing 193, 7-13 (2016)

14. Feng, Y, Yu, J, Li, C, Huang, T, Che, H: Linear impulsive control system with impulse time windows. J. Vib. Control 23(1), 111-118 (2017)

15. Feng, $Y, T u, D, L i, C$, Huang, $T$ : Uniformly stability of impulsive delayed linear systems with impulse time windows. Ital. J. Pure Appl. Math. 34, 213-220 (2015)

16. Li, C, Zhou, Y, Wang, H, Huang, T: Stability of nonlinear systems with variable-time impulses, B-equivalence method. Int. J. Control. Autom. Syst.

17. Yang, Z, Xu, D: Stability analysis and design of impulsive control systems with time delay. IEEE Trans. Autom. Control 52(8), 1448-1454 (2007)

18. Yang, $X$, Yang, Z, Nie, X: Exponential synchronization of discontinuous chaotic systems via delayed impulsive control and its application to secure communication. Commun. Nonlinear Sci. Numer. Simul. 19, 1529-1543 (2014)

19. Yang, X, Cao, J, Qiu, J: pth moment exponential stochastic synchronization of coupled memristor-based neural networks with mixed delays via delayed impulsive control. Neural Netw. 65, 80-91 (2015)

20. Yang, $X, L u$, J: Finite-time synchronization of coupled networks with Markovian topology and impulsive effects. IEEE Trans. Autom. Control 61(8), 2256-2261 (2016)

21. He, X, Huang, T, Yu, J, Li, C, Li, C: An inertial projection neural network for solving variational inequalities. IEEE Trans. Cybern. 47(3), 809-814 (2017)

22. Li, H, Liao, X, Huang, T, Zhu, W: Event-triggering sampling based leader-following consensus in second-order multi-agent systems. IEEE Trans. Autom. Control 60(7), 1998-2003 (2015)

23. Li, H, Chen, G, Huang, T, Dong, Z, Zhu, W, Gao, L: Event-triggered distributed average consensus over directed digital networks with limited communication bandwidth. IEEE Trans. Cybern. 46(12), 3098 (2016)

24. Li, H, Chen, G, Huang, T, Dong, Z: High performance consensus control in networked systems with limited bandwidth communication and time-varying directed topologies. IEEE Trans. Neural Netw. Learn. Syst. 28(5), 1043 (2017)

25. Li, H, Chen, G, Liao, X, Huang, T: Quantized data-based leader-following consensus of general discrete-time multi-agent systems. IEEE Trans. Circuits Syst. II, Express Briefs 63(4), 401-405 (2016)

26. Li, H, Liao, X, Chen, G, Dong, Z, Hill, D, Huang, T: Event-triggered asynchronous intermittent communication strategy for synchronization in complex networks. Neural Netw. 66, 1-10 (2015)

27. Li, H, Liao, X, Huang, T, Zhu, W, Liu, Y: Second-order globally nonlinear consensus in multi-agent networks with random directional link failure. IEEE Trans. Neural Netw. Learn. Syst. 26(3), 565-575 (2015)

28. $\mathrm{He}, \mathrm{X}, \mathrm{Li}, \mathrm{C}$, Huang, $T, \mathrm{Li}, \mathrm{C}$, Huang, J: A recurrent neural network for solving bilevel linear programming problem. IEEE Trans. Neural Netw. Learn. Syst. 25(4), 824-830 (2014)

29. Morimoto, T, Hawkes, E, Okamura, A: Design of a compact actuation and control system for flexible medical robots. IEEE Rob. Autom. Lett. 2(3), 1579-1585 (2017)

30. Zhang, L, Li, H: Evaluation system of urban environmental pollution based on the principal component analysis. In: 2016 International Conference on Smart City and Systems Engineering (ICSCSE)

31. Yu, D, Zheng, C, Iu, Ha, Fernando, T, Chua, L: A new circuit for emulating memristors using inductive coupling. IEEE Access 5, 1284-1295 (2017)

32. Huang, T, Li, C, Yu, W, Chen, G: Synchronization of delayed chaotic systems with parameter mismatches by using intermittent linear state feedback. Nonlinearity 22, 569-584 (2009)

33. Li, C, Liao, X, Yang, X, Huang, T: Impulsive stabilization and synchronization of a class of chaotic delay systems. Chaos 15, Article ID 043103 (2005) 
34. Feng, Y, Zou, L, Tu, Z: Stability analysis for a class of nonlinear impulsive switched systems. J. Nonlinear Sci. Appl. 10, 4544-4551 (2017)

35. Sanchez, E, Perez, J: Input-to-state stability (ISS) analysis for dynamic NN. IEEE Trans. Circuits Syst. I, Regul. Pap. 46(11), 1395-1398 (1999)

36. Boyd, S, El Ghaoui, L, Feron, E, Balakrishnan, V: Linear Matrix Inequalities in System and Control Theory. SIAM, Philadephia (1994)

37. Shilnikov, L: Chua's circuit: rigorous results and future problems. Int. J. Bifurc. Chaos 4(3), 489-519 (1994)

Submit your manuscript to a SpringerOpen ${ }^{\odot}$ journal and benefit from:

- Convenient online submission

- Rigorous peer review

Open access: articles freely available online

- High visibility within the field

- Retaining the copyright to your article

Submit your next manuscript at $\gg$ springeropen.com 